Applied Mathematical Sciences, Vol. 8, 2014, no. 116, 5783 - 5794

HIKARI Ltd, www.m-hikari.com

http://dx.doi.org/10.12988/ams.2014.47564

\title{
Risk Measures in Solvency Regulation: Reinsurance Layers and Unexpected Loss
}

\author{
Antonella Campana
}

Department S.E.Ge.S. University of Molise

Campobasso, Italy

\section{Paola Ferretti}

Department of Economics

Ca' Foscari University of Venice

Venice, Italy

Copyright (c) 2014 A. Campana and P. Ferretti. This is an open access article distributed under the Creative Commons Attribution License, which permits unrestricted use, distribution, and reproduction in any medium, provided the original work is properly cited.

\begin{abstract}
The total amount of damage to be paid by an insurance company can be represented as the sum of (reinsurance-)layers, some of which may be transferred to a reinsurer on the basis of an excess of loss treaty. Some risk measures associated with the layers are here investigated, by paying particular attention to the Unexpected Loss function which plays a role in the Solvency II legislative program: in fact in non-life insurance, the standard formula for the calculation of premium and the reserve capital requirement are mainly based on its definition. Starting from the assumption of discrete random variables, explicit formulas and ranges of variation are derived that are useful for its approximate evaluation.
\end{abstract}

Mathematics Subject Classification: 62P05

Keywords: non-proportional reinsurance, layers, risk measures, $V a R$, Unexpected Loss, Solvency II 


\section{Introduction}

In the framework of non-proportional reinsurance where it is assumed that the reinsurance protection deals with amounts above certain pre-defined limits, one main form is considered in literature: the excess of loss treaty.

The interest in reinsurance sector is mainly due to the intrinsic characteristic of each contract: in fact, ceding companies may increase the underwriting capabilities; net liability on individual risks may reduce; finally a catastrophe protection from multiple or large losses is practicable. As a consequence of the assortment of involved activities, reinsurance analysis reveals all its own complexity. Our main focus is to study risk measures for reinsurance layers because in this framework it is possible to represent the total claim as a sum of layers some of which are transferred to a reinsurer through an excess of loss treaty. Particular attention is devoted to the Unexpected Loss measure, which is considered in Solvency II program (see [4]), because in non-life insurance it constitutes a function for premium and reserve capital requirement computation. The paper is organized as follows. In Section (2) we first set the basic definitions for describing the problem of analyzing risk measures for reinsurance layers. Section (3) presents some results in the framework of excess of loss reinsurance involving the individual claim size or even the total claim amount. Section (4) is devoted to the problem of measuring how the studied risk measures change with reference to the particular assumption of discrete random variables associated to individual or total claim amount. Finally in Section (5) we focus our attention on Unexpected Loss function and upper and lower bounds are obtained that may be useful for its approximate evaluation. Section (6) ends the paper with some hints for future research.

\section{Preparatory settings and results}

Some notations, abbreviations and conventions used throughout the paper are the following. Hereafter, we will deal with real-valued random variables. More precisely, $F_{X}$ denotes the one-dimensional cumulative distribution function (cdf) of the random variable (r.v.) $X$, with $F_{X}(x)=\operatorname{Pr}[X \leq x]$, that is $F_{X}$ is a right-continuous (r.c.) non-decreasing function with

$$
F_{X}(-\infty)=\lim _{x \rightarrow-\infty} F_{X}(x)=0 \quad F_{X}(+\infty)=\lim _{x \rightarrow+\infty} F_{X}(x)=1
$$

In this paper we will refer to the usual definition of the inverse of a distribution function, that is for any real $k \in[0,1]$ we consider the inverse distribution function

$$
F_{X}^{-1}(k):=\inf \left\{x \in \mathbb{R}: F_{X}(x) \geq k\right\}
$$


where conventionally it is $\inf \emptyset=+\infty$. Note that it is possible to set a more sophisticated definition for the inverse of a distribution function in which a possible choice for the inverse of $F_{X}$ in $k$ is given by any point in the closed interval

$$
\left[\inf \left\{x \in \mathbb{R}: F_{X}(x) \geq k\right\}, \sup \left\{x \in \mathbb{R}: F_{X}(x) \leq k\right\}\right] .
$$

Clearly, if the cdf $F_{X}$ is increasing, then the two definitions collapse into the same one.

In algebra of inverse distribution functions, the following revealing result (see [2]) is set in order to compare the inverse distribution functions of the r.v. $X$ and of the transformed r.v. $g(X)$ through the monotone and left continuous (l.c.) function $g$.

Theorem 2.1 Let $X$ and $g(X)$ be real-valued random variables, and let $0<k<1$. If $g$ is non-decresing and l.c., then

$$
F_{g(X)}^{-1}(k)=g\left(F_{X}^{-1}(k)\right) .
$$

Again, when the inverse distribution function of a sum of random variables is considered, the comonotonic assumption (see [2] and [3]) plays a central role in ensuring that it is simply the sum of the inverse distribution functions of the marginal distributions.

Theorem 2.2 The inverse distribution $F_{S}^{-1}$ of a sum $S=X_{1}+X_{2}+\cdots+X_{n}$ of $n$ comonotonic random variables $X_{1}, X_{2}, \ldots, X_{n}$ is given by:

$$
F_{S}^{-1}(k)=\sum_{i=1}^{n} F_{X_{i}}^{-1}(k), \quad 0<k<1
$$

In the insurance framework, let $X$ now be a non-negative r.v. which denotes a claim amount. Moreover, let $g$ be a non-negative function, defined for all possible outcomes of $X$, which represents the benefit function, that is the reinsured amount. In this way the insurance company exposure to loss is reduced by passing part of the risk of loss to a reinsurer (or a group of reinsurers). A simple reinsurance contract can be represented by the risk sharing scheme, i.e. the pair of random variables $(g(X), X-g(X))$, where $g(X)$ denotes the amount promised by the reinsurer and $X-g(X)$ is the part of the claim covered by the ceding company. It is generally assumed that $g(X)$ and $X-g(X)$ are non-decreasing functions on the set of all the possible outcomes $x$ of $X$ : if the function $g$ is differentiable, non-decreasing monotonicity requirement is equivalent to the condition $0 \leq g^{\prime}(x) \leq 1$ for each outcomes $x$ of $X$. Given that both the partners of the risk sharing scheme $(g(X), X-g(X))$ have to bear more if the claim amount increases, then the random vector $(g(X), X-g(X))$ is comonotonic. On the converse, if the risk sharing scheme $(g(X), X-g(X))$ 
is supposed to be comonotonic, then necessarily there exist a support $\mathcal{A}$ of $X$ for which the set $\{(g(x), x-g(x)) \mid x \in \mathcal{A}\}$ is comonotonic. Accordingly, the two functions $g(x)$ and $x-g(x)$ must be necessarily monotonic of the same type on $\mathcal{A}$, that is they both display a non-decreasing or a non-increasing behavior. Given that the function $x=g(x)+(x-g(x))$ is non-decreasing, it must be that the functions $g(x)$ and $x-g(x)$ are both non-decreasing on $\mathcal{A}$.

The case of the risk sharing scheme $(g(X), X-g(X))$ results to be particularly interesting with reference to inverse distribution functions: in fact if $g$ is non-decresing and l.c. then by Theorem 2.1 it necessarily follows that

$$
F_{g(X)}^{-1}(k)=g\left(F_{X}^{-1}(k)\right) \quad F_{X-g(X)}^{-1}(k)=F_{X}^{-1}(k)-g\left(F_{X}^{-1}(k)\right), 0<k<1
$$

and by Theorem 2.2

$$
F_{X}^{-1}(k)=F_{g(X)}^{-1}(k)+F_{X-g(X)}^{-1}(k), 0<k<1 .
$$

\section{Reinsurance layer}

A very interesting example of risk sharing scheme is presented in the framework of excess of loss reinsurance where the r.v. $X$ represents the individual claim size or even the total claim amount. Now let us assume that the reinsured amount $g(X)$ is represented by the layer $X_{a, u, v}$ that is so defined

$$
X_{a, u, v}:=a \min \{v, \max (0, X-u)\}
$$

with $u \in[0, \infty), v \in(0, \infty)$ and $a \in(0,1]$. One may consider $u$ as a lower retention or deductible, $u+v$ as an upper retention or limit of coverage and $a$ represents a proportionality factor. The layer $X_{a, u, v}$ admits the following equivalent formalization (see [5])

$$
X_{a, u, v}=\left\{\begin{array}{ll}
0 & X \leq u \\
a(X-u) & u<X \leq u+v \\
a v & X>u+v
\end{array} .\right.
$$

The next lemma summarizes some interesting properties.

Lemma 3.1 The function $f(x)=x-a \min \{v, \max (0, x-u)\}$ where $u \in$ $[0, \infty), v \in(0, \infty)$ and $a \in(0,1]$, satisfies the following properties:

i) $f \in \mathcal{C}^{0}(\mathbb{R})$;

ii) $f \in \mathcal{C}^{k}(\mathbb{R} \backslash\{u, u+v\})$ for each index $k>0$;

iii) $f$ is monotone non-decreasing. 


\section{Proof}

The proof of properties $i$ ) and $i$ ) easily follows by $f$ definition: moreover, note that it is

$$
\lim _{x \rightarrow u} f(x)=u=f(u) \quad \lim _{x \rightarrow u+v} f(x)=(1-a) v+u=f(u+v) .
$$

With reference to property iii), let us recall that $f(x)=x-g(x)$ where $g(x)=$ $a \min \{v, \max (0, x-u)\}$ is evidently a non-decreasing function. Moreover, for each $x_{1}, x_{2}$ such that $x_{1}<x_{2}$ it is

$$
\begin{aligned}
\frac{f\left(x_{2}\right)-f\left(x_{1}\right)}{x_{2}-x_{1}} & =1-a \frac{\min \left\{v, \max \left(0, x_{2}-u\right)\right\}-\min \left\{v, \max \left(0, x_{1}-u\right)\right\}}{x_{2}-x_{1}} \\
& = \begin{cases}1, & x_{i} \in[0, u] \vee x_{i} \in[u+v,+\infty) ; \\
1-a, & x_{i} \in(u, u+v)\end{cases}
\end{aligned}
$$

where, in the last relation $x_{i}$ denotes $x_{1}$ or $x_{2}(i=1,2)$.

The following result involving the risk sharing scheme $\left(X_{a, u, v}, X-X_{a, u, v}\right)$ directly follows by Lemma 3.1 and (4):

Theorem 3.2 Given the non-negative random variable $X$, for each $u \in$ $[0, \infty), v \in(0, \infty)$ and $a \in(0,1]$, the risk sharing scheme $(g(X), X-g(X))$ with $g(x)=a \min \{v, \max (0, x-u)\}$ is a comonotonic random vector.

Comonotonicity of the risk sharing scheme $\left(X_{a, u, v}, X-X_{a, u, v}\right)$ ensure explicit formulation of some interesting quantities in non-proportional reinsurance framework that rely on inverse distribution functions of transformed random variables. By knowing the cdf $F$ of the r.v. $X_{a, u, v}$

$$
F_{X_{a, u, v}}(x)= \begin{cases}0 & x<0 \\ F_{X}\left(u+\frac{x}{a}\right) & 0 \leq x<a v \\ 1 & x \geq a v\end{cases}
$$

its inverse function may be easily defined

$$
F_{X_{a, u, v}}^{-1}(k)= \begin{cases}0 & 0<k \leq F_{X}(u) \\ a\left(F_{X}^{-1}(k)-u\right) & F_{X}(u)<k \leq F_{X}(u+v) \\ a v & F_{X}(u+v)<k<1\end{cases}
$$

Then, by theorems 3.2 and 2.2 it is possible to explicitly write the inverse distribution function of $X-X_{a, u, v}$; in fact it is

$$
F_{X-X_{a, u, v}}^{-1}(k)=F_{X}^{-1}(k)-F_{X_{a, u, v}}^{-1}(k), 0<k<1
$$


that is,

$$
F_{X-X_{a, u, v}}^{-1}(k)= \begin{cases}F_{X}^{-1}(k) & 0<k \leq F_{X}(u) \\ (1-a) F_{X}^{-1}(k)+a u & F_{X}(u)<k \leq F_{X}(u+v) . \\ F_{X}^{-1}(k)-a v & F_{X}(u+v)<k<1\end{cases}
$$

Inverse distribution function formulation is particularly interesting with reference to some very well-known risk measures as, for example, Value-atRisk. More precisely, given the Tail-quantile function $U_{X}$ associate to $F_{X}$ (see [5])

$$
U_{X}(q):=\inf \left\{x: F_{X}(x) \geq 1-\frac{1}{q}\right\}, \quad q>1
$$

the Value-at-Risk of a random variable $X$ with distribution function $F_{X}$ may be so defined

$$
\operatorname{VaR}_{q}(X):=U_{X}(q)=F_{X}^{-1}\left(1-\frac{1}{q}\right), \quad q>1 .
$$

Moreover, by referring to the Tail-quantile function it is possible to set the definition of a significant risk measure as the Unexpected Loss of the r.v. $X$ $U L_{X}$ (also called $V a R$-based economic capital in [1]) that has been considered in Solvency II project. It is defined as:

$$
U L_{X}(\epsilon)=F_{X}^{-1}(1-\epsilon)-E[X], \quad 0<\epsilon<1
$$

where

$$
F_{X}^{-1}(1-\epsilon)=\operatorname{VaR}_{\frac{1}{\epsilon}}(X)=U_{X}\left(\frac{1}{\epsilon}\right), \quad 0<\epsilon<1
$$

and $E[X]$ denotes the expected value of $X$ that in the following is supposed to be well-defined. In this way,

$$
U L_{X}(\epsilon)=U_{X}\left(\frac{1}{\epsilon}\right)-E[X], \quad 0<\epsilon<1 .
$$

In particular, with reference to a layer $X_{a, u, v}$, for each $q>1$ the Value-atRisk is

$$
\operatorname{VaR}_{q}\left(X_{a, u, v}\right)=\left\{\begin{array}{ll}
0 & 1<q<\frac{1}{1-F_{X}(u)} \\
a\left(F_{X}^{-1}\left(1-\frac{1}{q}\right)-u\right) & \frac{1}{1-F_{X}(u)}<q \leq \frac{1}{1-F_{X}(u+v)} \\
a v & q>\frac{1}{1-F_{X}(u+v)}
\end{array} .\right.
$$

Moreover, the Unexpected Loss can be easily obtained by referring to the following result

$$
F_{X_{a, u, v}}^{-1}(1-\epsilon)= \begin{cases}a v & 0<\epsilon<1-F_{X}(u+v) \\ a\left(F_{X}^{-1}(1-\epsilon)-u\right) & 1-F_{X}(u+v) \leq \epsilon<1-F_{X}(u) \\ 0 & 1-F_{X}(u) \leq \epsilon<1\end{cases}
$$


where $0<\epsilon<1$.

With reference to the r.v. $X-X_{a, u, v}$, the Unexpected Loss for $0<\epsilon<1$ is

$$
U L_{X-X_{a, u, v}}(\epsilon)=F_{X-X_{a, u, v}}^{-1}(1-\epsilon)-E\left[X-X_{a, u, v}\right]
$$

that is $($ see $(8))$

$$
U L_{X-X_{a, u, v}}(\epsilon)=F_{X}^{-1}(1-\epsilon)-F_{X_{a, u, v}}^{-1}(1-\epsilon)-E[X]+E\left[X_{a, u, v}\right]
$$

and so it is

$$
U L_{X-X_{a, u, v}}(\epsilon)=U L_{X}(\epsilon)-U L_{X_{a, u, v}}(\epsilon)
$$

\section{Layers and discrete random variables}

Starting from the assumption of random variables representing individual claim size, it is particularly interesting to analyze the special case of a combination of $N$ reinsured amounts $Y_{i}(i=1, \ldots, N)$ where each of them is supposed to be a layer $X_{a, u, v}$ : more precisely (see [5]), given the finite sequence $Y_{1 ; a, u, v}, \ldots, Y_{N ; a, u, v}$ of $N$ claims, where $N$ is a non-negative integer-valued random variable, let us consider the total claim amount

$$
R_{a, u, v}:=\sum_{i=1}^{N} Y_{i ; a, u, v}
$$

where $Y_{i ; a, u, v}=a \min \left\{v, \max \left(0, Y_{i}-u\right)\right\}$ stands for the reinsured part of the original claim size $Y_{i}$ defined for $i=1, \ldots, N$. Is is assumed that if $N \equiv 0$ then necessarily $R_{a, u, v} \equiv 0$.

In practice of insurance it is mostly assumed that a lower retention $U$ and a limit of coverage $U+V$ are applied to $R_{a, u, v}$; moreover, in many cases a proportionality factor $\alpha$ is considered. In this way the reinsured amount $S_{\alpha, U, V}$ is simply given by

$$
S_{\alpha, U, V}:=\alpha \min \left\{V, \max \left(0, R_{a, u, v}-U\right)\right\} .
$$

In particular, if it is assumed that each original claim $Y_{i}$ follows a discrete law with only two jumps (like the case of total loss), i.e.

$$
Y_{i} \stackrel{\mathrm{d}}{=} m B
$$

where $\stackrel{\mathrm{d}}{=}$ means equality in distribution, $m>0$ and $B$ denotes a Bernoulli random variable with

$$
\operatorname{Pr}[B=1]=p=1-\operatorname{Pr}[B=0]=1-q
$$


then the aggregate loss

$$
R:=\sum_{i=1}^{N} Y_{i}
$$

satisfies the equality in distribution

$$
R \stackrel{\mathrm{d}}{=} m N .
$$

By Theorem 2.1, the inverse distribution function of the r.v. $R$ is given by:

$$
F_{R}^{-1}(k)=m F_{N}^{-1}(k), 0<k<1
$$

where

$$
F_{N}^{-1}(k)=l \quad \text { if } \quad F_{N}(l-1)<k \leq F_{N}(l) .
$$

When $a=1, u=0$ and $v \leq m$, it is

$$
Y_{i ; 1,0, v} \stackrel{\mathrm{d}}{=} v B
$$

and

$$
R_{1,0, v}:=\sum_{i=1}^{N} Y_{i ; 1,0, v} \stackrel{\mathrm{d}}{=} v N .
$$

In the particular case in which $v>m$, it naturally follows that

$$
Y_{i ; 1,0, v} \stackrel{\mathrm{d}}{=} m B
$$

and

$$
R_{1,0, v} \stackrel{\mathrm{d}}{=} m N
$$

and this is why from now on we focus our attention on the case $v \leq m$. In fact it is generally supposed that the reinsurer agrees to indemnify the insurer for a proper subpart of the total loss.

If moreover there exist positive natural numbers $r$ and $s$ such that $U=r v$ and $V=s v, 0<r<s$, the reinsured amount $S_{\alpha, U, V}$ is

$$
S_{\alpha, U, V}=S_{\alpha, r v, s v}=\alpha \min \left\{s v, \max \left\{0, R_{1,0, v}-r v\right\}\right\}
$$

that is by $(23)$

$$
S_{\alpha, r v, s v}=\alpha v \min \{s, \max \{0,(N-r)\}\}
$$

that explicitly is

$$
S_{\alpha, r v, s v}=\left\{\begin{array}{ll}
0 & N \leq r \\
\alpha v(N-r) & r<N \leq r+s \\
\alpha s v & N>r+s
\end{array} .\right.
$$


The cdf of the r.v. $S_{\alpha, r v, s v}$ is given by

$$
F_{S_{\alpha, r v, s v}}(x)= \begin{cases}0 & x<0 \\ F_{N}\left(r+\frac{x}{\alpha v}\right) & 0 \leq x<\alpha s v \\ 1 & x \geq \alpha s v\end{cases}
$$

where $F_{N}$ denotes the cdf of the r.v. $N$. By Theorem 2.1 and (26), the inverse distribution function $F_{S_{\alpha, r v, s v}}^{-1}$ of the layer $S_{\alpha, r v, s v}$ is given by

$$
F_{S_{\alpha, r v, s v}}^{-1}(k)= \begin{cases}0 & F_{N}^{-1}(k) \leq r \\ \alpha v\left(F_{N}^{-1}(k)-r\right) & r<F_{N}^{-1}(k) \leq r+s \\ \alpha s v & F_{N}^{-1}(k)>r+s\end{cases}
$$

With reference to the Value-at-Risk computation of the layer $S_{\alpha, r v, s v}$ it is

$$
F_{S_{\alpha, r v, s v}}^{-1}(1-\epsilon)= \begin{cases}\alpha s v & 0<\epsilon<1-F_{N}(r+s) \\ \alpha v\left(F_{N}^{-1}(1-\epsilon)-r\right) & 1-F_{N}(r+s) \leq \epsilon<1-F_{N}(r) \\ 0 & 1-F_{N}(r) \leq \epsilon<1\end{cases}
$$

where $0<\epsilon<1$. Again, the layer expected value $E\left[S_{\alpha, r v, s v}\right]$ results so defined

$$
\begin{aligned}
E\left[S_{\alpha, r v, s v}\right] & =\alpha v E[\min \{s, \max (0,(N-r))\}] \\
& =\alpha v\left\{\sum_{n=r}^{r+s}(n-r) \operatorname{Pr}[N=n]+s \operatorname{Pr}[N \geq r+s]\right\} .
\end{aligned}
$$

\section{$5 \quad$ Unexpected Loss}

Let us focus our attention on the Unexpected Loss risk measure of the r.v. $S_{\alpha, r v, s v}$ in the particular case in which $\epsilon$ is bounded by the constraint $0<$ $\epsilon<1-F_{N}(r+s)$ : then the Unexpected Loss of the layer $S_{\alpha, r v, s v}$ admits the following representation

$$
\begin{aligned}
U L_{S_{\alpha, r v, s v}}(\epsilon) & =F_{S_{\alpha, r v, s v}}^{-1}(1-\epsilon)-E\left[S_{\alpha, r v, s v}\right] \\
& =\alpha v\left\{s \operatorname{Pr}[N \leq r+s-1]-\sum_{n=r}^{r+s}(n-r) \operatorname{Pr}[N=n]\right\}
\end{aligned}
$$

that is,

$$
U L_{S_{\alpha, r v, s v}}(\epsilon)=\alpha v\left\{s F_{N}(r+s-1)-\sum_{n=r+1}^{r+s}(n-r) \operatorname{Pr}[N=n]\right\}
$$

with no dependence from $\epsilon$. Given that $F_{N}(r+s-1) \leq F_{N}(r+s)<1-\epsilon$, it is possible to upper bound the Unexpected Loss of the layer $S_{\alpha, r v, s v}$

$$
U L_{S_{\alpha, r v, s v}}(\epsilon)<\alpha v\left\{s(1-\epsilon)-\sum_{n=r+1}^{r+s}(n-r) \operatorname{Pr}[N=n]\right\} .
$$


Let us consider the Unexpected Loss of the r.v. $R-S_{\alpha, r v, s v}$ representing the part of the claim covered by the ceding company

$$
U L_{R-S_{\alpha, r v, s v}}(\epsilon)=U L_{R}(\epsilon)-U L_{S_{\alpha, r v, s v}}(\epsilon)
$$

By assuming that $0<\epsilon<1-F_{N}(r+s)$, it is

$$
\begin{aligned}
U L_{R-S_{\alpha, r v, s v}}(\epsilon)= & m F_{N}^{-1}(1-\epsilon)-m E[N] \\
& -\alpha v\left\{s F_{N}(r+s-1)-\sum_{n=r+1}^{r+s}(n-r) \operatorname{Pr}[N=n]\right\}
\end{aligned}
$$

that admits the following equivalent formalization

$U L_{R-S_{\alpha, r v, s v}}(\epsilon)=m U L_{N}(\epsilon)-\alpha v\left\{s F_{N}(r+s-1)-\sum_{n=r+1}^{r+s}(n-r) \operatorname{Pr}[N=n]\right\}$

where $U L_{N}(\epsilon)$ denotes the Unexpected Loss of the r.v. $N$.

Note that the Unexpected Loss of the layer $S_{\alpha, r v, s v}$ doesn't explicitly depend on the choice of $\epsilon$ when it is assumed that it is small enough (here it is assumed that $\left.0<\epsilon<1-F_{N}(r+s)\right)$, while the Unexpected Loss of the part of the claim covered by the ceding company, $R-S_{\alpha, r v, s v}$, does depend on it.

With reference to this last case we move our attention to the study of upper and lower bounds. By (31) we have:

$$
U L_{R-S_{\alpha, r v, s v}}(\epsilon)>m U L_{N}(\epsilon)-\alpha v\left\{s(1-\epsilon)-\sum_{n=r+1}^{r+s}(n-r) \operatorname{Pr}[N=n]\right\} .
$$

By assuming that $F_{N}(r+s-1)>0$, from (33) it results

$$
U L_{R-S_{\alpha, r v, s v}}(\epsilon)<m U L_{N}(\epsilon)+\alpha v \sum_{n=r+1}^{r+s}(n-r) \operatorname{Pr}[N=n] .
$$

Finally, when it is assumed that $0<F_{N}(r+s-1)$ and $F_{N}(r+s)<1-\epsilon$, by setting

$$
\begin{aligned}
A & :=m U L_{N}(\epsilon)-\alpha v\left\{s(1-\epsilon)-\sum_{n=r+1}^{r+s}(n-r) \operatorname{Pr}[N=n]\right\} \\
B & :=m U L_{N}(\epsilon)+\alpha v \sum_{n=r+1}^{r+s}(n-r) \operatorname{Pr}[N=n]
\end{aligned}
$$

we obtain the following strict double inequality involving the Unexpected Loss of the claim section covered by the ceding company:

$$
A<U L_{R-S_{\alpha, r v, s v}}(\epsilon)<B
$$


Clearly it is

$$
A=B-\alpha v s(1-\epsilon)
$$

and when $\epsilon \rightarrow 0$ necessarily it follows that $A \rightarrow B-\alpha v s$.

If moreover $1-F_{N}(r+s+1) \leq \epsilon<1-F_{N}(r+s)$, then by (21) it results $F_{N}^{-1}(1-\epsilon)=r+s+1$. So it is

$$
\begin{aligned}
U L_{R-S_{\alpha, r v, s v}}(\epsilon)= & m(r+s+1)-m E[N] \\
& -\alpha v\left\{s \operatorname{Pr}[N \leq r+s]-\sum_{n=r+1}^{r+s}(n-r) \operatorname{Pr}[N=n]\right\}
\end{aligned}
$$

and by defining

$$
\begin{aligned}
C & :=m(r+s+1-E[N])-\alpha v\left\{s(1-\epsilon)-\sum_{n=r+1}^{r+s}(n-r) \operatorname{Pr}[N=n]\right\} \\
D & :=m(r+s+1-E[N])+\alpha v \sum_{n=r+1}^{r+s}(n-r) \operatorname{Pr}[N=n]
\end{aligned}
$$

the next double inequality descends when $1-F_{N}(r+s+1) \leq \epsilon<1-F_{N}(r+s)$ and $F_{N}(r+s-1)>0$ :

$$
C<U L_{R-S_{\alpha, r v, s v}}(\epsilon)<D
$$

In this case too

$$
C=D-\alpha \operatorname{se}(1-\epsilon)
$$

but $\epsilon \rightarrow 0$ only if $r+s \rightarrow \infty$ : then it necessarily follows $C \rightarrow D-\alpha v s$.

Note that given $m, v$ and $\alpha$, the difference between the sum $r+s$ and the expected number of claims $E[N]$ affects the value of $U L_{R-\alpha, R V, s v}(\epsilon)$. The quantities $v, r$ and $s$ define the extremes and the width of the layers and therefore they affect the risk measure.

\section{Concluding remarks}

Starting from the layer definition, in this paper we obtained and studied the explicit representation of some widely used risk measures, such as $V a R$ and Unexpected Loss, also for the purpose of exploring some solvency requirements as they are prescribed by the system Solvency II. The use of discrete probability distribution enabled to derive exact and approximate expressions of these special risk measures. This is not just a simplifying assumption: in fact it is often used in practice because the amounts of losses are always measured as multiples of a unit of measure. By referring to the obtained bounds it is possible to achieve approximate valuations of the Unexpected Loss function 
on the basis of which to make choices, in light of the capital requirements that are compulsory for the Solvency II system. This latter point is one of the addresses of our research.

\section{References}

[1] M.Denuit, J.Dhaene, M.J.Goovaerts and R.Kaas, Actuarial Theory for Dependent Risks: Measures, Orders and Models, John Wiley \& Sons, Chichester, 2005.

[2] J.Dhaene, M.Denuit, M.J.Goovaerts, R.Kaas and D.Vyncke, The concept of comonotonicity in actuarial science and finance: Theory, Insurance: Mathematics and Economics, 31 (2002), 3-33. http://dx.doi.org/10.1016/S0167-6687(02)00134-8

[3] J.Dhaene, M.Denuit, M.J.Goovaerts, R.Kaas and D.Vyncke, The concept of comonotonicity in actuarial science and finance: applications, Insurance: Mathematics and Economics, 31 (2002), 133-161. http://dx.doi.org/10.1016/S0167-6687(02)00135-X

[4] M.Eling, H.Schmeiser and J.T.Schmit, The Solvency II Process: overview and critical analysis, Risk Management and Insurance Review, 10 (2007), 69-85. http://dx.doi.org/10.1111/j.1540-6296.2007.00106.x

[5] S.Ladoucette and J.L.Teugels, Analysis of risks measures for reinsurance layers, Insurance: Mathematics and Economics, 38 (2006), 630-639. http://dx.doi.org/10.1016/j.insmatheco.2005.12.005

Received: July 4, 2014 